\title{
Efficiency of Open and Infill Trenches in Mitigating Ground-Borne Vibrations
}

Bose, Tulika; Choudhury, Deepankar; Sprengel, Julian; Ziegler, Martin

Published in:

Journal of Geotechnical and Geoenvironmental Engineering

Link to article, DOI:

10.1061/(ASCE)GT.1943-5606.0001915

Publication date:

2018

Document Version

Peer reviewed version

Link back to DTU Orbit

Citation (APA):

Bose, T., Choudhury, D., Sprengel, J., \& Ziegler, M. (2018). Efficiency of Open and Infill Trenches in Mitigating Ground-Borne Vibrations. Journal of Geotechnical and Geoenvironmental Engineering, 144(8), [04018048]. https://doi.org/10.1061/(ASCE)GT.1943-5606.0001915

\section{General rights}

Copyright and moral rights for the publications made accessible in the public portal are retained by the authors and/or other copyright owners and it is a condition of accessing publications that users recognise and abide by the legal requirements associated with these rights.

- Users may download and print one copy of any publication from the public portal for the purpose of private study or research.

- You may not further distribute the material or use it for any profit-making activity or commercial gain

- You may freely distribute the URL identifying the publication in the public portal 
1 Efficiency of open and infill trenches in mitigating ground-borne vibrations

\author{
Tulika Bose ${ }^{1}$, Deepankar Choudhury, M.ASCE², Julian Sprengel ${ }^{3}$, Martin Ziegler ${ }^{4}$
}

Abstract: In present-day context, man-made sources of ground-borne vibration are rising at a very rapid rate due to increasing construction works, blasting activities and rapidly expanding rail and road traffic system. As a consequence, amplified levels of ground-borne vibration occur, causing annoyance to residents living in nearby areas, posing a threat to the stability of old structures and interference with instrumentation works in industries. This paper aims to investigate the use of trenches, as a means of mitigation of ground vibration caused by propagation of surface (Rayleigh) waves. 2-D and 3-D finite element models have been developed using PLAXIS for identifying the key factors affecting the vibration isolation efficiency of open and infill trenches. Parametric studies have been carried out, and the results are analyzed to arrive at optimum values of geometrical and material properties of trenches. Numerical analysis shows that for open trenches, normalized depth is the decisive factor and width is of importance in case they are very shallow. For infill trenches, it is observed that lowdensity materials perform exceedingly well as infill materials, but their performance is highly sensitive to the relative shear wave velocity between infill material and the in-situ soil. Finally, as a particular case of infill trenches, an in-depth study has been carried out to investigate the performance of geofoam trenches in mitigating vibrations caused by a harmonic load. In addition, the analysis has been extended to bring forth the effectiveness of these geofoam barriers in damping out the vibrations generated by a moving train. In this case, it is noted that the barrier efficiency increases with an increase in train speed. The key findings suggest that trenches could prove to be a simple and effective solution for reducing ground-borne vibrations. 
$26{ }^{1}$ Ph.D. Research Scholar, Department of Civil Engineering, Technical University of Denmark,

27 DK-2800 Kgs. Lyngby, DENMARK. And, former PG student, Department of Civil

28 Engineering, Indian Institute of Technology Bombay, Mumbai 400076, INDIA. Email:

29 bosetulika11@gmail.com

$30{ }^{2 *}$ Institute Chair Professor, Department of Civil Engineering, Indian Institute of Technology

31 Bombay, Powai, Mumbai 400076, INDIA. Also Adjunct Professor, Academy of Scientific and

32 Innovative Research (AcSIR), CSIR Campus, INDIA. E-mail: dc@civil.iitb.ac.in

$33{ }^{3}$ Ph.D. Research Scholar, Department of Geotechnical Engineering, RWTH Aachen

34 University, Aachen 52074, GERMANY.Email: sprengel@geotechnik.rwth-aachen.de

$35{ }^{4}$ Professor and Director of Geotechnical Engineering and Institute of Foundation Engineering,

36 Soil mechanics, Rock mechanics and Waterways construction, RWTH Aachen University,

37 Aachen 52074, GERMANY. Email: ziegler@geotechnik.rwth-aachen.de

38

39 CE Database subject headings:

40 Geofoam trenches, Infill trenches, Moving loads, Numerical finite element model, Open 41 trenches, Vibration isolation, Wave barriers. 
Introduction

43

Ground-borne vibrations generated from machines, construction activities and transportation sector are often of intense levels, posing a great challenge for engineers to build structures in such areas, which can be serviceable to the residents. The problem has become acute with the advent of high-speed railway networks, expanding at a very rapid rate across the globe. As the train speeds and axle loads keep on increasing, the vibration levels are amplified to a great extent. Energy from the surface sources of vibration mainly propagates in the form of Rayleigh waves, which are confined to a narrow zone near to the surface of the elastic half space (Choudhury and Katdare 2013). In addition, these waves attenuate with distance in a rather slow manner when compared to the Body waves, which predominates near to the source of vibration. Hence, vibration induced damages and distress to structures on the surface are extremely high on account of the Rayleigh waves (Choudhury et al. 2014).

Vibration isolation using trenches (open or infill) as wave barriers, can be an ideal solution as they might be a quick, simple and cost- competitive way to deal with this problem. Trenches function as wave barriers by curbing the motion of the travelling wave, leading to degeneration of energy. An open trench acts like a finite discontinuity in the ground surface across which no energy is transmitted. For an infill trench, there is a difference in material impedance at the junction of in-situ soil and the trench. This causes energy redistribution across the trench in the form of reflected and transmitted waves. Trenches are used as wave barriers in two different scenarios- i) Active or near-field isolation and ii) Passive or far-field isolation. In the former case, they are built enclosing the source of vibration, like vibrating machines, etc., while the latter is built near to the objects to be shielded, like buildings to be protected from vibrations of nearby rail and road traffic.

In vibration isolation studies involving wave barriers, numerical method of analysis has found more popularity. The theoretical solutions are limited in number, involving simplified 
geometries, while the full scale testing methods are often expensive to perform. One of the earliest experimental works were done by Barkan (1962), Neumeuer (1963), and Mc Neil at al. (1965). Not all these attempts proved to be successful but findings from these works, gave insight into the mechanism of screening by wave barriers. Woods (1968) performed a series of field experiments for both near and far-field isolation using open trenches. A minimum trench depth of $0.6 L_{R}$ and $1.33 L_{R}\left(L_{R}=\right.$ Rayleigh wavelength) was suggested for active and passive isolation respectively, considering 75\% screening efficiency. Haupt (1981) carried out a number of scaled model tests using both solid concrete barriers as well as lightweight bore holes and open trenches. The study showed that the efficiency of the barrier was a function of the parameters in terms of wavelength normalized dimensions. Aboudi (1973), Fuyuki and Matsumoto (1980), and, May and Bolt (1982) carried out numerical studies using FEM or FDM. Later, BEM found popularity owing to its simplicity and was widely used in wave propagation problems, e.g. Emad and Manolis (1985), Beskos et al. (1986), and Leung et al. (1987). An extensive parametric study was carried out by Ahmad and Al Hussaini (1991) on use of open and infill trenches as wave barriers. The screening efficiency for open trenches was found to be dependent mainly on depth, while, for infill trenches, it was reported to be a function of both depth and width. Al-Hussaini and Ahmad (1991) studied horizontal screening efficiency of barriers and reported that trenches were more effective in damping out vertical vibrations in comparison to horizontal vibrations. Ahmad et al. (1996) used 3-D BEM to study active isolation of machines using open trenches, while, Al-Hussaini and Ahmad (1996) used infill trenches for the same purpose. The results were compared with experimental works and a good agreement was found between the two. Yang and Hung (1997) developed a finite element model with infinite elements to investigate efficiency of open and infill trenches due to passage of trains. It was reported that trenches were less effective in screening the low frequencies of vibration. Hung et al. (2004) carried out similar studies and observed that 
trenches were more effective in screening waves caused by a train moving at supercritical speed compared to subcritical speed. Ju and Lin (2004) also reported similar results. Adam and Estorff (2005) employed a coupled BEM-FEM in time domain to study the effectiveness of trenches in reducing building vibrations. They found that $80 \%$ of the forces in the building component could be reduced by a well-designed barrier. Andersen and Nielsen (2005) applied the same coupled approach and reported that trenches proved to be a better solution to mitigate vertical vibrations compared to horizontal vibrations. Wang (2006, 2009) numerically investigated the efficiency of EPS barriers in protecting buried structures under blast load. Leonardi and Buonsanti (2014) studied efficiency of concrete and compacted soil barriers for reducing train induced vibrations. Esmaeili et al. (2014) and Zakeri et al. (2014) carried studies on V shaped and step shaped trenches respectively. Their findings revealed that trenches with such modified geometries were more effective compared to conventional rectangular trenches. Full scale experimental studies were conducted by Massarsch (1991) to study the efficiency of gas cushion screen systems which were found to be comparable with open trenches. Baker (1994) carried out field tests on stiffer and softer barriers made of concrete and bentonite respectively. Davies (1994) carried out 20g centrifuge tests to study the screening effectiveness of EPS barriers on buried objects. The studies indicated that low acoustic materials could reduce the magnitude of ground shock loading on buried structures. Zeng et al. (2001) performed tests on rubber modified asphalt and found that owing to a high damping ratio, it could be used effectively beneath high speed railway tracks as a foundation material, for vibration attenuation. Itoh et al. (2005) conducted centrifuge tests and suggested using a combination of crumb rubber modified asphalt at the vibration source and an EPS barrier along the transmission path. Murillo et al. (2009) performed 50g centrifuge tests on EPS barrier and reported the incremental efficiency as a function of depth of the barrier. Alzawi and EI Naggar (2011) carried out full scale field tests to study effectiveness of geofoam barriers. Their findings 
revealed that significant increase in performance could be observed for normalized barrier depth greater than 0.6.

The research carried till date lacks a systematic procedure of selecting the best infill material which can be used in trenches for a given soil domain. Most of the studies have been carried out for open trenches or with selected infill materials like concrete or bentonite. In addition, the loading has mostly been considered to be harmonic in nature and the trench geometry to be a rectangular single wall type. A generalized performance of materials based on their characteristic properties like density or stiffness is missing in the literature. There is also a need for determining the sensitivity of trench efficiency to the change in each of the individual geometrical parameters of the trenches. In this study, an attempt has been made to bridge in this gap by studying the performance of trenches methodically, beginning with the simplest case of open trenches. Then, parametric studies are carried out over a wide range of materials which can be used in an infill trench for a given soil domain and their relative effectiveness are compared. Later, the efficiency of a geofoam barrier system is investigated in depth for both a harmonic load as well as for a moving train. The objective of this study is to study the performance of trenches in a holistic manner for various materials, different geometrical parameters, system configurations and various loading applications.

2-D and 3-D numerical finite element models are developed using PLAXIS. The developed numerical models are validated with the works of previous authors and then used to carry out studies on open and infill trenches. Parametric variation of material and geometrical properties of the infill trenches is carried out and comparative analysis of the efficiency are presented in all cases. The optimum barrier dimensions are also highlighted. In this study, the soil is considered to be elastic, homogenous and isotropic. The loading considered is initially periodic and harmonic in nature and later has been modified to simulate a moving train. 
A numerical model is developed to understand the behaviour and efficiency of open trenches, as wave barriers, in mitigating ground-borne vibrations generated due to a harmonic load vibrating in the vertical direction. The 2-D axisymmetric model consists of 15 noded triangular elements. The average element size is fixed based on the recommendations given by Kuhlemeyer and Lysmer 1973, following which the mesh size should be 1/8 - 1/10 of the wavelength. In order to account for the semi-infinite extent of the soil, viscous boundary conditions are assigned along the model edges so as to avoid undue wave reflections. Standard fixities are applied, wherein, the vertical sides are restrained horizontally $\left(u_{x}=0\right)$ and the bottom is fully restrained $\left(u_{x}=u_{y}=0\right)$. A linear elastic soil model is chosen as wave propagation problems in soil involving trenches usually generate small strains. Therefore, the material nonlinearities arising due to the small variations of the stress over a cycle will not be very influential. Considering this, at the small strain levels, the soil behaviour can be assumed to be linearly elastic without significant loss of accuracy. (Yang and Hung 1997; Andersen and Nielsen 2005; Alzawi and EI Naggar 2011). In wave propagation problems involving barriers, in order to avoid any dependency of the results on the frequency of the load, the geometrical parameters of the trench are usually normalized with those of the Rayleigh wavelength, $L_{R}$ (Ahmad and Al-Hussaini 1991). Figs. 1 and 2 represent the schematic view and the meshing details of the developed numerical model respectively.

\section{Validation of present model}

The results of any vibration isolation scheme are typically expressed in the form of amplitude reduction ratio, $A R R$ (Woods 1968), which is given as per Eq. (1) as:

$$
A R R=\frac{A_{I}}{A_{O}}
$$


The values of $A R R$ vary at different locations beyond the trench. To have an idea of the overall $A R R$ over the barrier influence zone $(x)$. This is represented by the parameter, average amplitude reduction ratio ( $A r$ ), given by Eq. (2):

$$
A r=\frac{1}{x} \int(A R R) d x
$$

From this, the overall system efficiency or effectiveness ( $E f$ ) is evaluated using Eq. (3):

$$
E f=(1-A r) * 100
$$

The numerical model is first validated with the works of previous researchers. For that purpose, an open trench of depth, $d=1.0 L_{R}$, width, $w=0.1 L_{R}$, and screening distance, $l=5 L_{R}$ is considered. Fig. 3a shows a plot of the variation of amplitude reduction ratio, $A R R$ with normalized distance beyond the source of vibration. A good agreement is found between the simulated results and those reported by the earlier authors.

To compute the system efficiency as a whole, the average amplitude reduction is to be calculated over a zone of influence, $x$ beyond the barrier. For this purpose, the extent of area over which the trench exerts its influence is determined by plotting the normalized soil particle displacement beyond the barrier, as shown in Fig. 3b. It is evident from this plot that after a distance of roughly $10 L_{R}$ beyond the open trench, the particles displacements are fairly insignificant and the influence of the trench almost diminishes. Hence, to enumerate $\operatorname{Ar}, x=$ 
For an open trench, there are three variables: depth $(d)$, width $(w)$ and screening distance $(l)$, which can be optimized to achieve the maximum screening efficiency. In this study, the trench is placed at different locations and corresponding to each position, a wide combination of width and depth are chosen and the system efficiency is evaluated. The input parameters for the soil domain are as per Yang and Hung 1997. The relevant properties are: density, $\rho=1800 \mathrm{~kg} / \mathrm{m}^{3}$, shear wave velocity, $V_{S}=101 \mathrm{~m} / \mathrm{sec}$, Rayleigh wave velocity, $V_{R}=93 \mathrm{~m} / \mathrm{sec}, L_{R}=3 \mathrm{~m}$, Poisson's ratio, $v=0.25$, and, damping coefficient, $\xi=5 \%$. The source of vibration is taken to be a periodic harmonic load of magnitude $1 \mathrm{kN}$ vibrating vertically at a frequency of $31 \mathrm{~Hz}$. For practical purpose, the footing carrying the vibrating load is not included in the numerical model as it does not alter or affect the results of the study (Kattis et al. 1999).

Fig. 4 demonstrates the results of the parametric study, plotted in terms of variation of average amplitude reduction ratio/system efficiency with a change in normalized geometrical parameters of the trench. The barrier is placed at two particular screening distances $(L=3$ and 5), and analyzed for a wide range of depth $(D)$ and width $(W)$. It is observed that open trenches have an excellent vibration isolation capacity. In the range considered for the parametric study here, the minimum efficiency of the system is as high as $55 \%$, while the maximum ranges to more than $80 \%$. It becomes evident that the normalized depth is the key parameter controlling the system effectiveness. The efficiency is maximized with the increase in normalized depth, $D$. This is true for all the chosen locations and widths of the trench. To have an efficiency $E f>60 \%$, the normalized depth, $D$ should be greater than 0.8 . In addition, it is noted that the performance of the trench is not very sensitive to the barrier location, $L$. The same is true for the trench width, $W$, with the exception of very shallow trenches. For these cases, the response of the system improves with an increase in width. This is mainly due to the fact that open trenches are discontinuity in the ground profile across which no part of the wave energy is 
allowed to pass and so, wave reflection plays the major role. Hence, for a sufficiently deep barrier which is able to obstruct the Rayleigh waves, creation of a finite discontinuity in the ground surface is enough. However, for a very shallow trench the situation changes, as in this case, not the entire wave energy is blocked by the barrier and so width has an important role to play. This observation is consistent with the findings reported previously by Ahmad and AlHussaini 1991.

\section{$\underline{\text { Vibration isolation efficiency of infill trenches }}$}

2-D parametric study

Open trenches, though an excellent solution for mitigating ground-borne vibrations, find their use in limited cases owing due to stability issues. Hence, infill trenches become a popular choice when the wavelength exceeds a depth, beyond which open vertical cuts find difficulty in construction and stability. For an open trench, wave reflection plays the major role, while, for an infill trench it is the combination of energy in the reflected and transmitted waves that governs its efficiency.

In this study, a wide array of infill materials are chosen having different densities ( $\left.\rho_{\text {fill }}\right)$, both lower as well as higher compared to the soil domain. For each material density, the shear wave velocity ( $\left.V_{\text {Sfill }}\right)$ is gradually increased from low to high values. A parametric study is carried out with these widespread spectra of infill materials and their relative efficiencies are assessed. Also, for a few chosen densities, variations of the damping characteristics of the materials are also performed. The relevant parameters considered for the soil domain are: $\rho_{\text {soil }}$

$233=1850 \mathrm{~kg} / \mathrm{m}^{3}, V_{\text {Ssoil }}=225 \mathrm{~m} / \mathrm{sec}, V_{\text {soil }}=0.4, \xi_{\text {soil }}=5 \%$. The dynamic load is simulated to be periodic and harmonic in nature, vibrating vertically at a frequency of $45 \mathrm{~Hz}$. The barrier is has been varied from 0.02 to 4.20 . The shear wave velocity ratio of infill material to that of the 
soil, $V_{\text {Sfill }} / V_{\text {Ssoil }}$ has been changed from 0.25 to 6.0. The damping properties of the infill have been kept in the range of 5\% $-15 \%$.

Results of parametric study

Fig. 5 shows a plot, depicting variation of $A r$ with a change in shear wave velocity ratio of infill to that of the soil, for various material density ratios. It is observed that the functioning of the trench is largely dependent on the contrast between the properties of the infill and the in-situ soil. Both density and shear wave velocity of the infill material has a great impact on the obtained results. The behaviour can be described separately for low and high density materials.

In general, low-density materials, $\rho$ fill $/ \rho_{\text {soil }}<0.15$ perform really well as wave barriers compared to dense materials. In fact, their performance can be comparable to open trenches. However, their response depends on the relative shear wave velocity between the infill material and the in-situ soil, $V_{\text {sfill }} / V_{\text {Ssoil. }}$ The system performs efficiently, displaying lower Ar values, when the shear wave velocity of the fill material is lower compared to soil. This happens because in this case the low density materials have sufficient energy dissipation capacity. With increase in shear wave velocity of fill, the Ar values start to increase or the efficiency decreases. An upper limiting value can be identified as1; as $V_{\text {Sfill }} / V_{\text {Ssoil }}$ approaches 1.0 the $A r$ values show a very sharp increase.

On the other hand, dense infill materials, $\rho$ fill $/ \rho_{\text {soil }}>1.0$ can also function effectively in the trench, depicting lower $A r$ values. This happens when they have a very high shear wave velocity compared to in-situ soil. The lower limit in this case can be identified to be 2.5; for $V_{\text {sfill }} / V_{\text {ssoil }}>2.5$, the values of $A r$ are generally lower or efficiency is higher. It indicates that high density materials having sufficient stiffness are able to resist the incoming wave. Materials having density in the range of $\left(0.15<\rho_{\text {fill }} / \rho_{\text {soil }}<1\right)$ perform well, when the shear wave velocity lies in the range $\left(1.0>V_{S f i l l} / V_{\text {Ssoil }}>2.5\right)$. 
Fig. 6 shows the variation of $A r$ with change in damping properties of the infill materials, keeping all other parameters unchanged. It is observed that the values of $A r$ are not very sensitive to the changes in the damping characteristics of the infill materials. With a variation of damping coefficient of the infill from 5\%-15\%, no significant changes were detected in the values of $\mathrm{Ar}$.

\section{Vibration isolation efficiency of geofoam trenches}

The results in the previous section indicate that low density materials having lower shear wave velocity relative to the surrounding soil domain are ideal as infill materials to be used in the trenches. Following that, further analyses have been carried out by selecting a low density geofoam material to be used in the trenches. The type of geofoam used is Polyurethane. It is a leading member of the wide range and diverse family of polymers, manufactured both in solid as well as cellular forms and can be rigid as well as flexible.

\section{A. 2-D Parametric study}

A step-wise sensitivity analysis is carried out to investigate the relative influence of all the relevant geometrical parameters of the Polyurethane Foam (PU-Foam) trench on its efficiency. At first, the impact of geofoam trench depth, $D$ and screening distance, $L$, are analyzed while keeping the width to be constant at $0.25 \mathrm{~m}$. Following that, the width of the trench, $w$ is varied for chosen depths, keeping the trench fixed at two particular locations, simulating near field and far field isolation. Finally, the influence of the cross-sectional area, $A$ and ratio of $d / w$ is investigated. The soil and loading parameters remain same as taken for the infill trenches. The properties of the PU-Foam used are taken from Alzawi and EI Naggar (2011) as: $V_{S}=$ $330 \mathrm{~m} / \mathrm{sec}, \rho=61 \mathrm{~kg} / \mathrm{m}^{3}$.

Results and discussions

(i) Influence of depth and location of the barrier 
The trench is placed at different locations, $L$ and at each position, the normalized depth, $D$ is changed from 0.3 to 1.5 , while the width remains constant. Fig. 7a represents the combined influence of $D$ and $L$ on average amplitude reduction ratio, $A r$ of the geofoam trenches. Firstly, it is observed that PU-foam trenches have a very good vibration isolation capacity. The $\mathrm{Ar}$ values show a major dependency on both the screening distance and the normalized depth. Secondly, it is observed that on changing the normalized distance, $L$ from 0.4 to $1.6, A r$ changes in a complex manner depending on the depth. For $L>1.8$, the effectiveness is mostly governed mainly by normalized depth and is almost independent of the location of the barrier from the source of vibration. So, it can be said that increasing $D$, generally results in reduction in $A r$, can be considered to be around 1.2 for all practical purposes.

\section{(ii) Influence of width of barrier}

In this case, the trench is placed at two different locations. In the first case, it simulates relatively near-field isolation, with $L=0.4$ and for the second case, it is far-field isolation, with $L=1.5$. For both the cases, simulations are performed for a few chosen depths, by varying the width of the trench while keeping the barrier location fixed. Figs. $7 \mathrm{~b}$ and $7 \mathrm{c}$ illustrates the influence of width, $w$, on average amplitude reduction ratio, $A r$ of the geofoam trenches. It can be clearly seen that unlike an open trench, where width does not play any significant role in the system-efficiency; here substantial impact of width on the performance is observed. This occurs because, for an infill trench, wave reflection, absorption and transmission, all have a role to play and hence, the stiffness of the system is important as a whole, in which the width 
is an integral part. As an example, it can be shown that with an increase in width from 0.15 to 0.35, the $\mathrm{Ar}$ values decrease by about $40 \%$ for almost all the chosen depths and for both the cases. Also from this figure, it becomes evident that for near-field isolation (Fig. 7b) the increase of depth does not have the same impact on the system efficiency as compared to farfield isolation (Fig. 7c).

(iii) Influence of cross-sectional area and slenderness ratio of the barrier

For a particular location of the barrier at $L=1.5$, the influence of cross sectional area, $A$ on the performance of the trench is investigated along-with determination of optimum $d / w$ ratio for each cross sectional area. The cross sectional area of the trench, $A$ is increased gradually from $0.5 \mathrm{~m}^{2}$ to $2.5 \mathrm{~m}^{2}$. For each area, the slenderness of the trench $(\mathrm{d} / \mathrm{w})$ is progressively varied from 0.5 to around 6.0. Fig. $7 d$ demonstrates the combined influence of cross sectional area and slenderness ratio on the functioning of the trenches. It is observed for lower cross sectional areas, $\left(A<1.0 \mathrm{~m}^{2}\right)$, it is reasonable to construct a deeper trench $(d / w=4.0-5.0)$ than a shallow one to have greater efficiency. However, for larger cross sectional areas, $\left(A>1.0 \mathrm{~m}^{2}\right)$, it is sufficient to construct a trench having $d / w$ in the range of 1.5-2.0. Extra cost incurred in creating deeper trenches does not bring about greater benefits. In fact, with increase in cross sectional area the optimum $d / w$ ratio hovers near 1.5. Again, for low slenderness ratio values, $d / w<2$, the increase in cross-sectional area has a very positive impact on the efficiency of the system. For the particular case when $d / w=1$, the $A r$ values decrease by about $55 \%$ when $A$ is increased from $0.5 \mathrm{~m}^{2}$ to $2.5 \mathrm{~m}^{2}$. For higher values, $d / w>2$, the increase in area does not have much of an impact on the performance of the system except for very small cross sections like $A<0.80 \mathrm{~m}^{2}$.

\section{B. 3-D finite element model and analysis}

336

\section{Validation of present model}


337 After an extensive 2-D parametric study on performance of PU-Foam trenches as wave barriers, a 3-D analysis is carried out for studying their responses with other configurations and loading conditions. A 3-D finite element model (100 m x 50 m x 20 m) is developed in PLAXIS 3D with its dynamic module using 10 noded tetrahedral elements. The model dimensions are chosen in a manner so as to avoid any boundary effects (Kumar et al. 2017, Kumar and Choudhury 2018). Viscous boundaries are applied along the edges so as to account for the semi-infinite extent of the soil and prevent undue reflection of the waves along the boundaries (Kumar et al. 2015, 2016). Literature studies show that the wave relaxation coefficients related to absorbent boundaries, taken to be $C 1=1.0$ and $C 2=0.25$ results in a reasonably good absorption of the waves at the edges (Wang et al. 2009; Brinkgreve and Vermeer 1998). Accordingly these values are adopted in the present study. The boundary conditions involve (i) completely restraining the bottom edge and, (ii) restricting the vertical model boundaries from moving in the direction of their normal. The element size is kept roughly less than $1 / 8^{\text {th }}$ of the smallest Rayleigh wavelength (Kuhlemeyer and Lysmer 1973). In addition, local refinement of the mesh is done near the critical areas of interest like loading zone, barrier location and in general on the ground surface to ensure high degree of accuracy of the results. Fig 8a depicts the discretized 3-D model developed for this problem. A linear elastic model is adopted for all the materials considering a small strain behaviour.

The numerical model is first validated with the works of previous researchers. For this purpose, the field data recorded by Alzawi and EI Naggar (2011) is taken for comparison. Fig. 8b shows a good agreement between the results obtained in this study and those observed by Alzawi and EI Naggar (2011). The differences noted in some cases could be due to variability and anisotropy in soil properties in localized areas in the field.

\section{Results and discussions}

(i) Influence of the type of barrier system 
The 3-D model is next employed to study the response of other configurations of the geofoam barrier. The most common profile adopted for wave barriers involves a straight, rectangular vertical cut into the ground. In this section, calculations are performed with another simple barrier configuration, involving two continuous foam walls kept at a spacing (s); depicted pictorially in Fig. 9a. Simulations are carried out to determine the influence of the spacing (s) between the two walls on the system performance. Analysis are performed by varying the normalized spacing $\left(S=s / L_{R}\right)$ from 0.2 to 1.0 . The computations are done for the frequency range of $30 \mathrm{~Hz}$ to $60 \mathrm{~Hz}$. The PU-Foam barriers are of normalized depth, $D=0.75$, width, $w=$ $0.2 \mathrm{~m}$ and placed at $L=0.53 \mathrm{~m}$ from the source of vibration. The size, fixities, boundary conditions and meshing of the 3-D model remains same as described in the preceding section. The material properties of the in-situ soil and geofoam remain unchanged. The values of $A r$ are computed for vertical velocity component, by observing the time history of nodes on the ground surface along a monitoring path. Fig. 9b illustrates the variation of the average amplitude reduction ratio as a function of the barrier normalized spacing for the chosen frequency range. It can be easily noted that this barrier system is quite effective in damping out the vertical soil vibrations. The $A r$ values are much lower at higher frequencies, indicating a better system performance. In addition, the system response is quite sensitive to the barrier spacing, especially at low values of $S(S=0.2$ to 0.4$)$. In the frequency range chosen for this study, the optimum spacing is obtained to be around $0.5 L_{R}$ to $0.6 L_{R}$. Wider spacing than this, does not bring any added benefits in terms of increase in efficiency and are even detrimental to the performance in some cases.

\section{(ii) Barrier performance for a moving load}

\section{Simulation of moving load}


Until now, a harmonic load was considered in all the analyses. However, the scenario is changed when the load apart from being dynamic in nature also shifts its position with time, which is the case for a moving load like a train. A moving load can significantly increase displacements in the structure compared to a static load. Thus, the response of the PU-Foam barrier is investigated under the vibrations generated by moving loads.

Fig. 10 shows the developed numerical model. It has dimensions of $200 \mathrm{~m} \mathrm{x} 100 \mathrm{~m} \mathrm{x}$ $20 \mathrm{~m}$. The dimensions have been kept large enough so as to prevent wave reflections from the boundaries. The track rests on an embankment of width $5 \mathrm{~m}$ and height $0.5 \mathrm{~m}$. For simplicity, the properties of the soil in the embankment and the ground remains same (as described previously). The track consists of a pair of steel rails resting on concrete sleepers; both modelled using beam elements. The cross-sectional area $(A)$ of the rail and the sleeper are: $A_{\text {rail }}$ $=0.0077 \mathrm{~m}^{2}$ and $A_{\text {sleeper }}=0.05 \mathrm{~m}^{2}$. The sleepers are laid on ground at a spacing $(c)$ of $0.6 \mathrm{~m}$.

The vehicle unit chosen for this demonstration is a typical German ICE3 railcar with the distance $(X)$ between the first and last wagon axles as $21.6 \mathrm{~m}$. The length of the loading in the rail $(X o)$ was chosen to be: $X o=X+2 * 0.3 X \approx 34.80 \mathrm{~m}$; the additional length to account for shear force distribution and effects of impact load distribution (Shahraki et al. 2014). To replicate a train moving on the rails, point loads are applied along the length of the beam at spacing of: $c / 2=0.30 \mathrm{~m}$. Thus, the total number of dynamic loads $(N)$ per rail are: $N=X_{o} /$ $c / 2=117$. The value assigned to each point load is the vertical wheel load $(P=80 \mathrm{kN})$. To incorporate the moving nature of the load, dynamic signals/multipliers are assigned to each of these 117 point loads. The signal for each load location represents how the forces vary at that particular point in the rail as the wheel load moves along. The multipliers are obtained by considering the rail to be a beam resting on an elastic pin foundation and analyzed under a set of unit static loads at different locations. Each point load is multiplied by the value of its own signal for every time dynamic time step. The latter is the parameter which accounts for the time 
taken by the train to cross a distance of $c / 2$. As an example for a train travelling at a speed $(V)$ of $180 \mathrm{~km} / \mathrm{hr}$, the time lag between two consecutive point loads are : $\Delta t=c / 2 / V=0.3 \mathrm{~m} / 50 \mathrm{~m} / \mathrm{s}$ $=0.006 \mathrm{sec}$. Accordingly, the time step for this dynamic analysis is kept as $0.006 \mathrm{sec}$. The total time of analysis is based on the time taken by the last axle to cross the loading zone. In this study, analyses have been carried out for train speeds $250 \mathrm{~km} / \mathrm{hr}, 180 \mathrm{~km} / \mathrm{hr}$ and $80 \mathrm{~km} / \mathrm{hr}$. At these chosen speeds, the track responses can be assumed to be mainly quasi-static and the dynamic effects to be negligible. The dynamic forces due to wheel-rail irregularity and other defects due to wheel-flats are not part of this study. Here, the focus is on quasi-static track response. The geofoam trench is chosen to be of depth $5 \mathrm{~m}$ and width $0.5 \mathrm{~m}$. It has been placed at roughly $10 \mathrm{~m}$ from center of railway track. The material properties of the foam and the soil domain remain same as before.

Results and discussions

Fig. 11a demonstrates the influence of train speed on the velocity of soil particles on the ground surface. It is seen that with increase in train speed, the velocity of vibration increases, especially in the near field region. This is most notably marked for vertical vibrations. The vertical vibration levels are very high in the near field condition but their attenuation with distance occurs at a very fast rate. At distances far away from the source of vibration, the horizontal and the vertical velocities show nearly same values for all the train speeds. Fig. 11b presents a set of typical results of the analysis in the frequency domain. It compares the velocity of vibration for the different speeds in absence of trench. It is observed that with an increase in the train speed, the frequency of ground vibration increases. For train speed of $80 \mathrm{~km} / \mathrm{hr}$, the frequency of vibration ranges from $0-30 \mathrm{~Hz}$. For speed of $180 \mathrm{~km} / \mathrm{hr}$, the predominant range of vibration is $10-40 \mathrm{~Hz}$, while for a speed of 250km/hr, the range extends from 20-55 Hz. Fig. 12 compares the frequency of vertical vibration, in presence and absence of trench, for the different train speeds. From Fig. 12a, it is clearly observed that for the train speed of $80 \mathrm{~km} / \mathrm{hr}$, the frequency 

of vibration, post-trench installation is mostly arrested within $20 \mathrm{~Hz}$. The frequencies in the zone $20-30 \mathrm{~Hz}$ are partly damped by the wave barrier. The same trend is noted in Figs. $12 \mathrm{~b}$ and 12c. In the former case (Fig 12b), the frequency of vibration in the zone of $30-40 \mathrm{~Hz}$, is mostly damped out. The particles vibrate primarily in the range of 0-30 Hz, especially, within 1020Hz. In the latter case (Fig 12c), the frequencies higher than $40 \mathrm{~Hz}$ are completely blocked by the barrier. This shows that the high frequency or shorter wavelength waveforms are blocked very effectively by the barrier. For the chosen barrier depth $(5 \mathrm{~m})$ and soil profile, corresponding to a frequency of $40 \mathrm{~Hz}$, the normalized depth $D$ is approximately 1.0 . For frequencies higher than $40 \mathrm{~Hz}$, the value of $D$ is greater than 1 and the barrier effectively depletes these frequency contents. Hence, more efficiency is achieved at higher train speeds as in this case the quasi-static track response has higher frequency contents.

\section{Conclusions}

A numerical finite element analysis was carried out using PLAXIS, to interpret the behaviour of open and infill trenches, acting as wave barriers in scaling down the ground vibration levels caused by surface sources. The study brings forth the behaviour and responses of trenches for a wide range of geometrical and material properties, different barrier types and loading conditions. The analysis was carried out in stages, from open cuts to infill ones, with special focus on polyurethane foam trenches. The developed model was used to carry out a parametric study in order to identify the key factors affecting the vibration isolation capacity of trenches. 2-D simulations were performed in order to understand the impact of geometrical and material properties of the trenches, on its efficiency as a wave barrier, due to vibrations caused by a harmonic load. Subsequently, 3-D analysis was carried out when the load apart from being dynamic in nature also changed its position with time. 
- Open trenches performed exceedingly well in mitigating the ground vibrations. The main parameter controlling the efficiency was the normalized depth, $D$. For $D>0.8$, the system effectiveness in all cases, irrespective of the location or the width, was found to be greater than $75 \%$. Width of the trench did not play a very important role except for extremely shallow trenches. The efficiency of an open trench as wave barrier was not very sensitive to screening distances.

- For infill trenches, the most important parameters governing the efficiency was: density and shear wave velocity of the infill material relative to the in-situ soil. The damping characteristics of the infill material did not have a significant impact on the efficiency of the trenches much. Both low-density and high-density materials (in comparison to in-situ soil) could be ideal for use in infill trenches but, their performance was highly sensitive to the relative stiffness of the trench material and the in-situ soil. For the former category $\left(\rho_{\text {fill }} / \rho_{\text {soil }}<0.15\right)$ the upper limit could be identified as $V_{\text {sfill }} / V_{\text {Ssoil }}<$ 1.0; whereas for the latter ( $\rho_{\text {fill }} / \rho_{\text {soil }}>1$ ), the lower limit was found to be $V_{\text {Sfill }} / V_{\text {ssoil }}>$ 2.5 .

- PU-Foam trenches proved to be very effective material in damping out the groundborne vibrations. The efficiency of the geofoam trenches was dependent on the normalized depth, width, screening distance, and, $d / w$ ratio. In areas near to the source of vibration, $(0.4<L<1.8)$ the barrier showed a greater dependency on both the screening distance and the depth, while, in regions far away, $(L>1.8)$ the influence of screening distance was almost eliminated. The optimum barrier depth for all purposes could be taken as 1.2. The increase in width had a positive impact on the functioning of the barrier both in near field as well as far-field isolation. On considering crosssectional areas; for $A<1.0 \mathrm{~m}^{2}$, a deeper trench $(d / w=4.0-5.0)$ served a greater purpose. However, for $A>1.0 \mathrm{~m}^{2}$, the optimum $d / w$ ratio was in the range of 1.5-2.0. 
- 3-D analysis revealed that double walled continuous rectangular trenches performed well as wave barriers but the functioning was sensitive to the normalized spacing. An optimum normalized spacing in this study was recognized to be roughly 0.5-0.6 times the Rayleigh wavelength.

- The barriers were also found to be quite effective in damping out the vibrations caused by passage of a moving load. They mostly damped out the high frequency or shorter wavelength components from the vibration velocities; indicating an increase in efficiency of the system, with an increase in train speed.

These observations can be generalized to arrive at the conclusion that trenches can prove to be very effective when used as a wave barrier in mitigating ground-borne vibrations.

\section{Acknowledgment}

The first author would like to thank the German Academic Exchange Service (DAAD), for providing financial assistance to carry out a part of the study at RWTH Aachen University, Germany under the DAAD-IIT Masters Students Exchange Program.

\section{References}

Aboudi, J. (1973). "Elastic waves in half-space with thin barrier." Journal of Engineering Mechanics, ASCE, 99(1), 69-83.

Adam, M., and Von Estorff, O. (2005). "Reduction of train-induced building vibrations by using open and filled trenches.” Computers and Structures, 83 (1), 11-24.

Ahmad, S., and Al-Hussaini, TM. (1991). "Simplified design for vibration screening by open and in-filled trenches.” Journal of Geotechnical Engineering, ASCE, 117(1), 67-88. 
508 Ahmad, S., Al-Hussaini, T.M., and Fishman, K.L. (1996). "Investigation on Active Isolation 509 of Machine Foundations by Open Trenches.” Journal of Geotechnical Engineering, ASCE, 510 122(6), 454-461.

511 Al-Hussaini, T.M., and Ahmad, S. (1991). "Design of Wave Barriers for Reduction of 512 Horizontal Ground Vibration.” Journal of Geotechnical Engineering, ASCE, 117(4), 616-636.

513 Al-Hussaini, T.M., and Ahmad, S. (1996). “Active Isolation of Machine Foundations by In514 Filled Trench Barriers.” Journal of Geotechnical Engineering, ASCE, 122(4), 288-294.

515 Alzawi, A., and El Naggar, M.H. (2011). "Full scale experimental study on vibration scattering 516 using open and in-filled (geofoam) wave barriers.” Soil Dynamics and Earthquake 517 Engineering, 31, 306-317.

518 Andersen, L., and Nielsen, SRK. (2005). "Reduction of ground vibration by means of barriers 519 or soil improvement along a railway track.” Soil Dynamics and Earthquake Engineering, 25, $520701-716$.

521 Andersen, L., and Jones, C.J.C.(2006). “Coupled boundary and finite element analysis of 522 vibration from railway tunnels—a comparison of two-and three-dimensional models”. Journal 523 of sound and vibration, 293(3), 611-625.

524 Baker, J.M. (1994). “An experimental study on vibration screening by in-filled trench barriers.” M.Sc. thesis, State University of New York, Buffalo, USA. Brinkgreve B.J., Vermeer, P.A. (1998) “PLAXIS finite element code for soil and rock analysis”. Delft, the Netherlands: A. A. Balkema Publishers.

528 Barkan, D. D. (1962). Dynamics of Bases and Foundations. Mac Graw-Hill Book Company 529 Inc., 374-406. Beskos, D. E., Dasgupta, G., and Vardoulakis, I. G. (1986). “Vibration isolation using open or 
532 Choudhury, D. and Katdare, A. D. (2013). "New approach to determine seismic passive

533 resistance on retaining walls considering seismic waves.” International Journal of

534 Geomechanics, 13(6), 852-860.

535 Choudhury, D., Katdare, A. D. and Pain, A. (2014). "New method to compute seismic active 536 earth pressure on retaining wall considering seismic waves.” Geotechnical and Geological 537 Engineering, An International Journal, 32(2), 391-402.

538 Davies, M.C.R. (1994). “Dynamic soil-structure interaction resulting from blast loading.” 539 Proceedings of the International Conference on Centrifuge Modelling - Centrifuge, 94, 319540324.

541 Emad, K., and Manolis, G. D. (1985). Shallow trenches and propagation of surface waves. 542 Journal of Engineering Mechanics, ASCE, 111(2), 279-282.

543 Esmaeili, M., Zakeri, J., and Mosayebi, S. (2014). Investigating the Optimized Open V-Shaped 544 Trench Performance in Reduction of Train-Induced Ground Vibrations. International Journal 545 of Geomechanics, 14 (3), 4-11.

546 Fuyuki, M., and Matsumoto, Y. (1980). "Finite difference analysis of Rayleigh wave scattering 547 at a trench." Bull. Seismol. Soc. Am., 70(6), 2051-2069.

548 Haupt, W.A. (1981). "Model tests on screening of surface waves.” In: Proceedings of the tenth 549 international conference on soil mechanics and foundation engineering, Stockholm, 3, 215550222.

551 Hung, H.H., Yang, Y. B., and Chang D. W. (2004). "Wave Barriers for Reduction of Train552 Induced Vibrations in Soils.” Journal of Geotechnical and Geoenvironmental Engineering, 553130 (12), 283-291.

554 Itoh, K., Zeng, X., Koda, M., Murata, O., and Kusakabe, O. (2005). “Centrifuge simulation of 555 wave propagation due to vertical vibration on shallow foundations and vibration attenuation 556 countermeasures.” Journal of Vibration and Control, 11, 781-800. 
Ju, S.H., and Lin, H.T. (2004). “Analysis of train-induced vibrations and vibration reduction

558

559

560

561

562

563

564

565

566

567

568

569

570

571

572

573

574

575

576

577

578

579

580

581

schemes above and below critical Rayleigh speeds by finite element method.” Soil Dynamics and Earthquake Engineering, 24, 993-1002.

Kattis, S.E., Polyzos, D., and Beskos, D.E. (1999). "Vibration Isolation by a Row of Piles Using a 3-D Frequency Domain BEM.” International Journal of Numerical Methods in Engineering, 46, 713-28.

Kramer SL. (1996). “Geotechnical Earthquake Engineering.” Prentice-Hall Inc.,Upper Saddle River, NJ, USA.

Kuhlemeyer, R.L., and Lysmer, J. (1973). "Finite element method accuracy for wave propagation problems.” Journal of Soil Mechanics and Foundation Division, 99, 421-427.

Kumar, A. and Choudhury, D. (2018). “Development of new prediction model for capacity of combined pile-raft foundations.” Computers and Geotechnics, 97, 62-68.

Kumar, A., Choudhury, D., and Katzenbach, R. (2016). "Effect of earthquake on combined pile-raft foundation.” International Journal of Geomechanics, 16(5), 1-16.

Kumar, A., Choudhury, D., Shukla, J. and Shah, D. L. (2015). "Seismic design of pile foundation for oil tank by using PLAXIS3D.” Disaster Advances, 8(6), 33-42.

Kumar, A., Patil, M. and Choudhury, D. (2017). “Soil-structure interaction in a combined pileraft foundation - a case study.” Proceedings of the Institution of Civil Engineers - Geotechnical Engineering, 170(2), 117-128.

Leonardi, G., and Buonsanti, M. (2014). "Reduction of Train-induced Vibrations by using Barriers.” Research Journal of Applied Sciences, Engineering and Technology, 7 (17), 623632.

Leung, K. L., Vardoulakis, I. G., and Beskos, D. E. (1987). "Vibration isolation of structures from surface waves in homogeneous and nonhomogeneous soils." Soil-structure interaction, A. S. Cakmak, ed., Elsevier, Amsterdam, The Netherlands, 155-169. 
Massarsch, K.R. (1991). “Ground vibration isolation using gas cushions.” Proceedings of the 2nd International Conference on Recent Advances in Geotechnical Earthquake Engineering and Soil Dynamics, St. Louis, MO, University of Missouri at Rolla, Rolla, MO, 1461-1470. May, T. W., and Bolt, B. A. (1982). “The effectiveness of trenches in reducing seismic motion.” Earthquake Engineering and Structural Dynamics, 10 (2), 195-210.

McNeill, R. L., Margason, B. E., and Babcock, F. M. (1965). "The role of soil dynamics in the design of stable test pads." Proc, Guidance and Control Conference,366-375.

Murillo, C., Thorel, L. and Caicedo, B. (2009). "Ground vibration isolation with geofoam barriers: Centrifuge modeling.” Geotextiles and Geomembranes, 27, 423-34.

Neumeuer, H. (1963). “Untersuchungen uber die Abschirmung eines bestehenden Gebaudes gegen Erschutterungen beim Bau and Betrieb einer U-Bahnstrecke.”Baumaschine and Bautechnik-10, Jahrgang, Heft 1, 23-29 (in German).

Richart, F.E., Hall, J.R. and Woods, R.D. (1970). “Vibrations of Soils and Foundations.” Prentice-Hall Inc., Upper Saddle River, NJ, USA.

Shahraki, M., Sadaghiani, M.R.S., Witt, K.J., and Meier, T. (2014). “3D Modelling of Train Induced Moving Loads on an Embankment”, Plaxis Bulletin Issue 36, Autumn.

Wang, J.G., Sun, W., and Anand, S. (2009). "Numerical investigation on active isolation of ground shock by soft porous layers.” Journal of Sound and Vibration, 321, 492-509.

Wang, Z.L., Li, Y.C., and Wang, J.G. (2006). “Numerical analysis of attenuation effect of EPS geofoam on stress-waves in civil defense engineering.” Geotextiles and Geomembranes, 24, 265-273.

Woods, R.D. (1968). "Screening of Surface Waves in Soils.” Journal of Soil Mechanics and Foundation Engineering Division, ASCE, 94 (4), 951-79. 
605 Yang, Y.B., and Hung, H.H. (1997). “A Parametric Study of Wave Barriers for Reduction of 606 Train Induced Vibrations.” International Journal for Numerical Methods in Engineering, 40, $607 \quad 3729-47$.

608 Zakeri, Jabbar-Ali, Esmaeili, M. and Mosayebi, Seyed-Ali.(2014). "Numerical investigation of 609 the effectiveness of a step-shaped trench in reducing train-induced vibrations." Proceedings of 610 the Institution of Mechanical Engineers, Part F: Journal of Rail and Rapid Transit, 228 (3), $611 \quad 298-306$.

612 Zeng, X., Rose, J.G., and Rice, J.S. (2001). “Stiffness and damping ratio of rubber modified 613 asphalt mixes: potential vibration attenuation for high speed railway track beds.” Journal of $614 \quad$ Vibration and Control, 7 (4), 527-538.

615 


\begin{tabular}{|c|c|}
\hline$\xi$ & Damping coefficient \\
\hline$v$ & Poisson's ratio \\
\hline$\Delta t$ & Dynamic time step \\
\hline$\rho$ & Density \\
\hline$d$ & Depth of trench \\
\hline$f$ & Frequency \\
\hline l & Distance \\
\hline$S, C$ & spacing \\
\hline$w$ & Width of trench \\
\hline$\overline{A R R}$ & Amplitude reduction ratio \\
\hline$A r$ & Average amplitude reduction ratio \\
\hline$A$ & Cross-sectional area \\
\hline$D$ & Normalized depth of the trench \\
\hline$E$ & Elastic modulus \\
\hline$G$ & Shear modulus \\
\hline$L$ & Normalized distance \\
\hline $\bar{L}$ & Rayleigh wavelength \\
\hline$N$ & Number of dynamic loads \\
\hline$P$ & Vertical wheel load \\
\hline$S$ & Normalized spacing \\
\hline$V_{R}$ & Rayleigh wave velocity \\
\hline$V_{S}$ & Shear wave velocity \\
\hline$W$ & Normalized width of the trench \\
\hline Xo & Axle distance in rail-cars \\
\hline
\end{tabular}





\section{Figures}

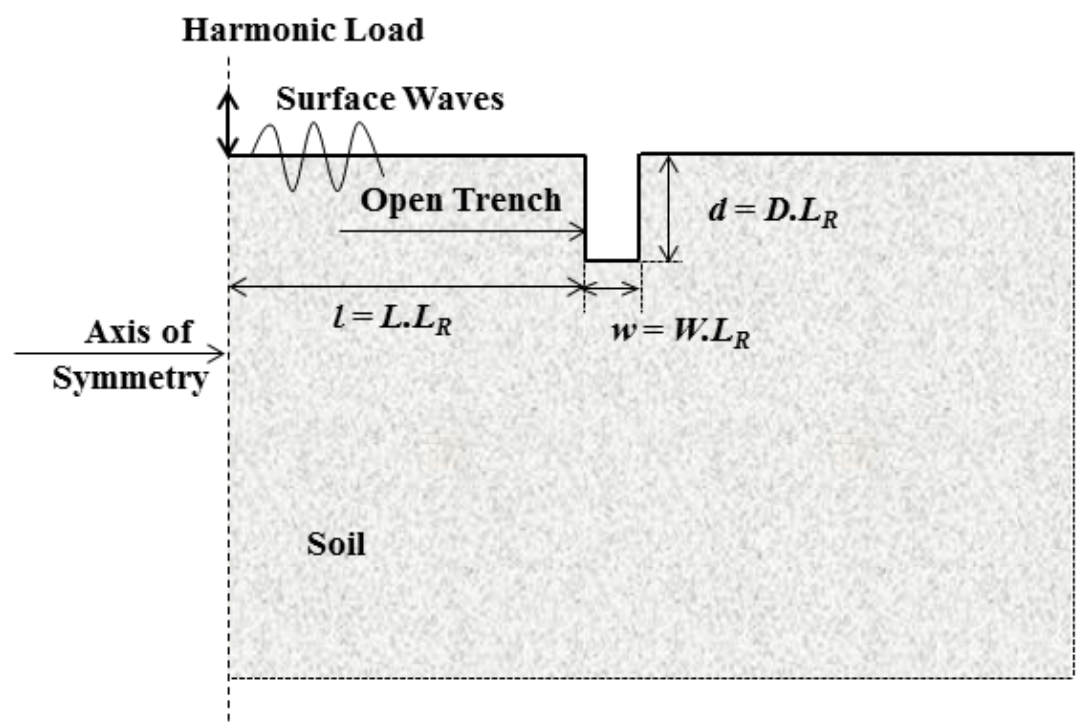

Figure 1: Schematic representation of vibration isolation system using open trench

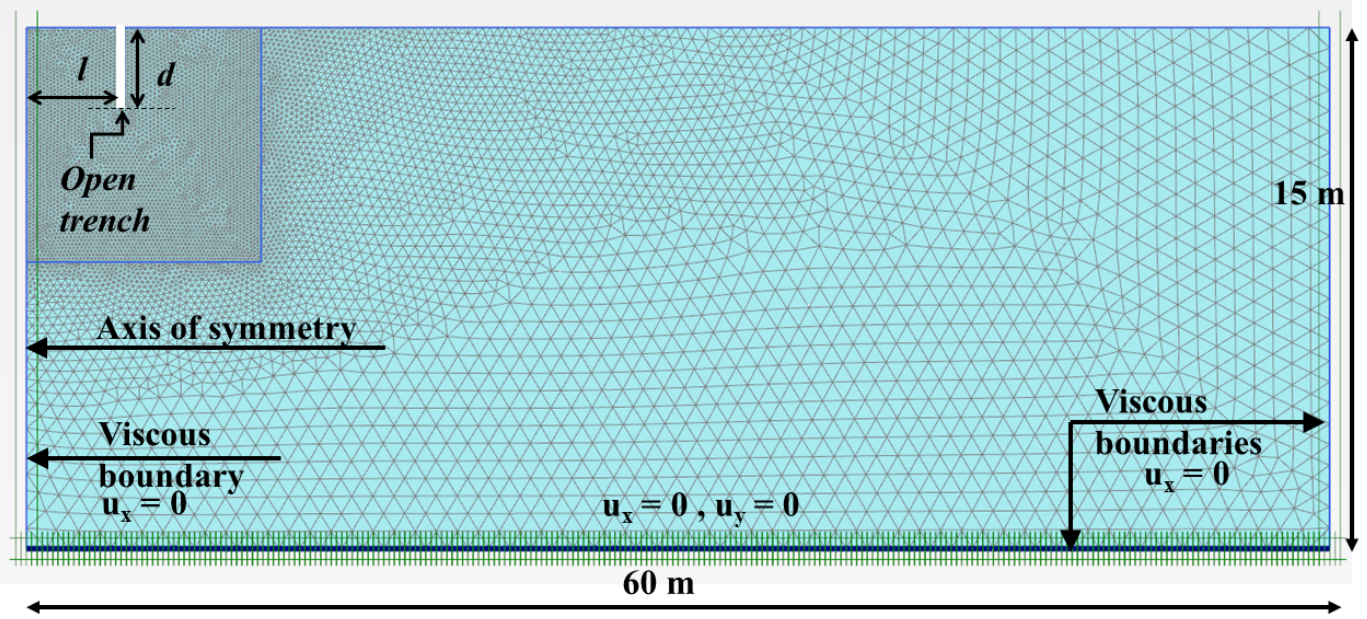

Figure 2: Typical 2-D numerical model developed for open trench in PLAXIS 


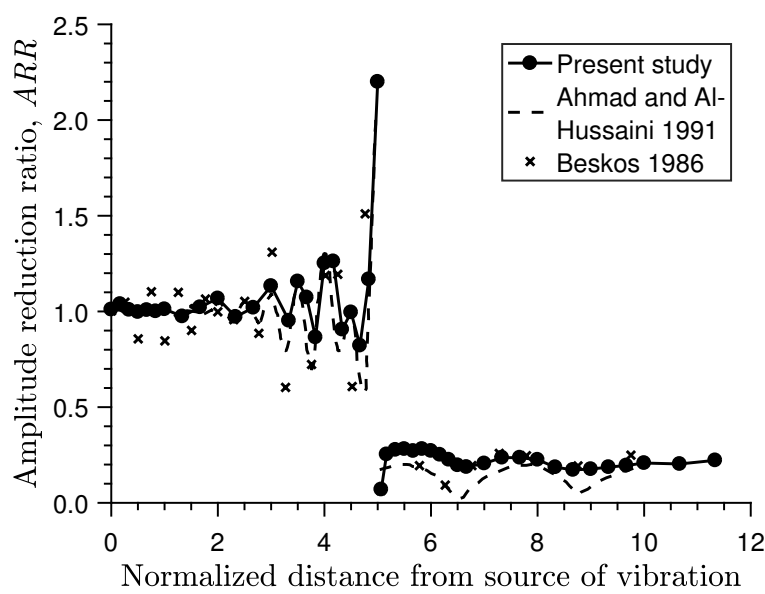

(a) Model validation

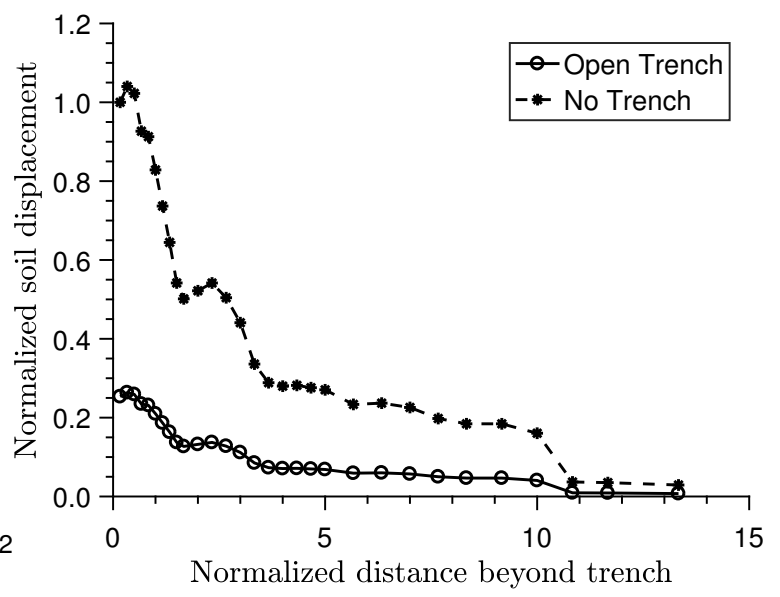

(b) Trench influence zone

Figure 3: Analysis of open trench (a) 2-D finite element model verification, $W=0.1, D=1, L$ $=5$, and (b) normalized vertical displacement amplitude of ground surface

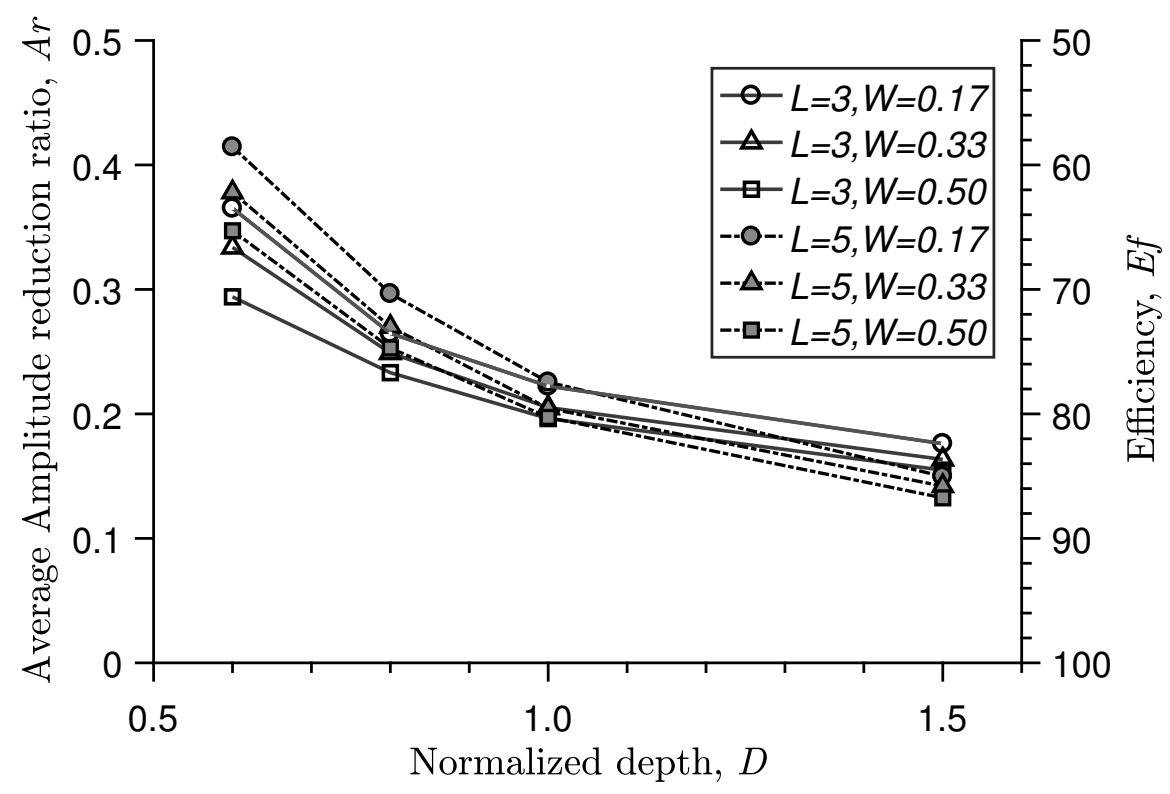

Figure 4: Variation of average amplitude reduction ratio with change in normalized depth of open trench 


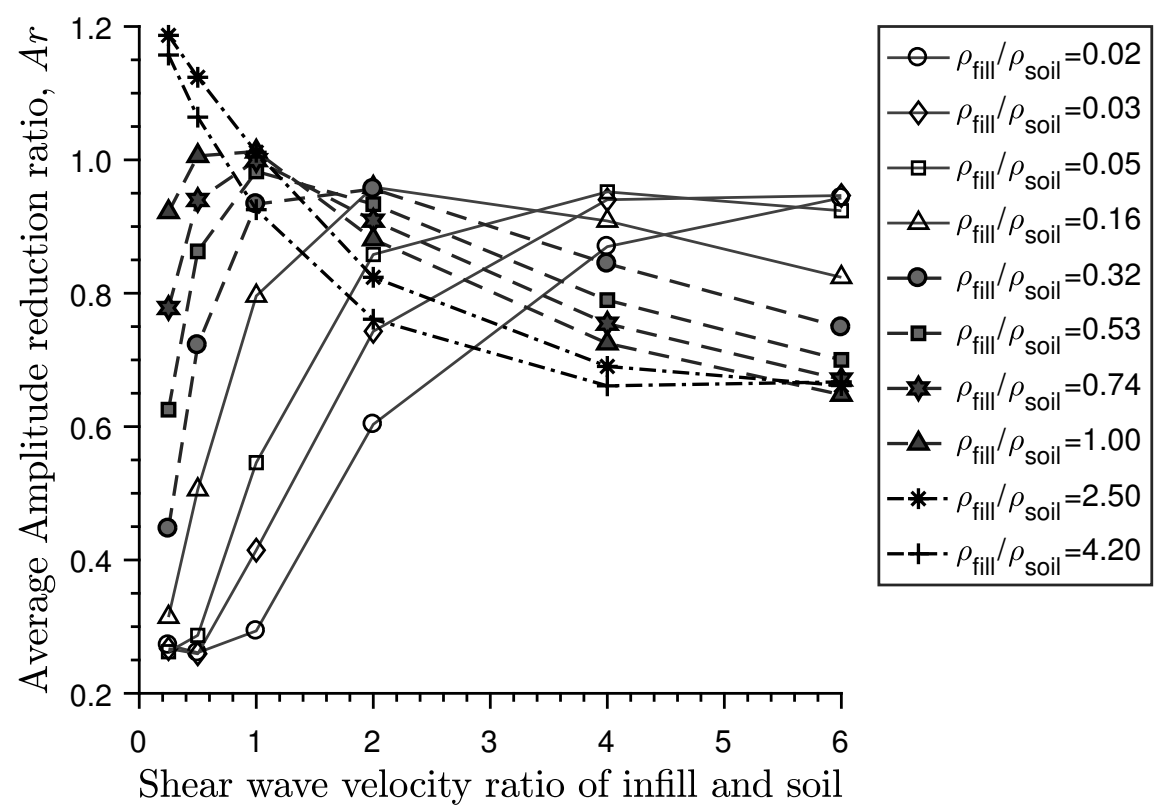

Figure 5: Variation of average amplitude reduction ratio with change in shear wave velocity ratio of infill trench and in-situ soil

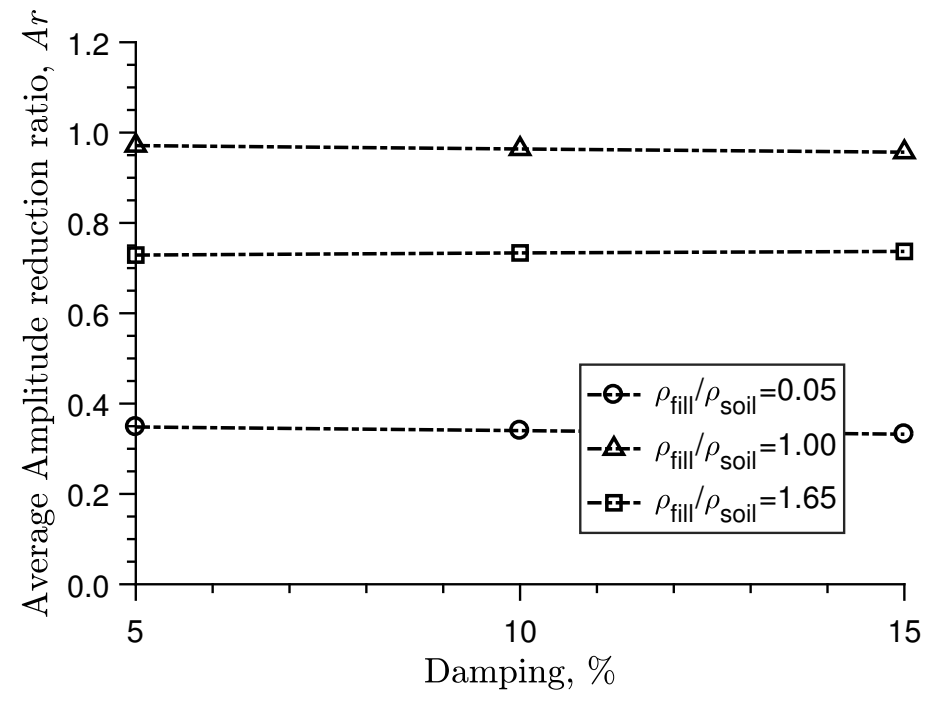

Figure 6: Variation of average amplitude reduction ratio with change in damping characteristics of infill trench 


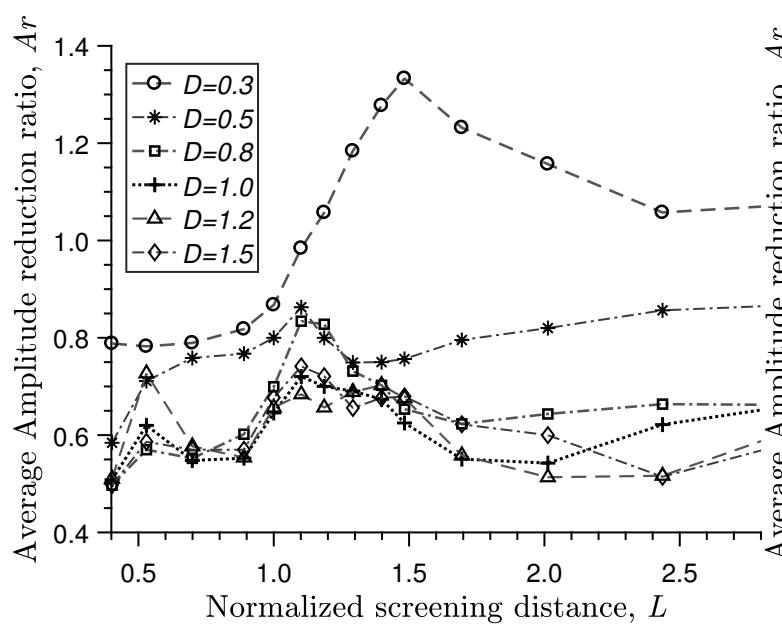

(a) $\operatorname{Ar}$ vs $L$

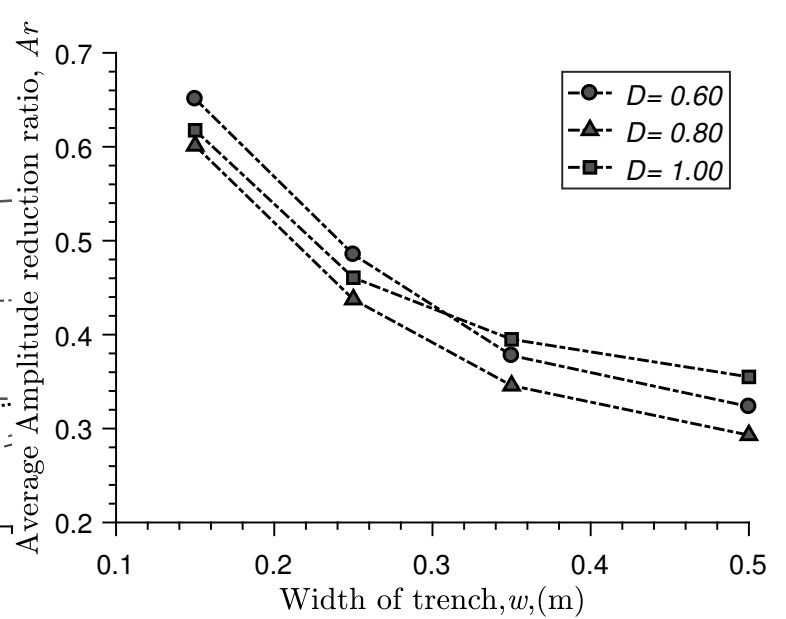

(b) $A r$ vs $w, L=0.4$

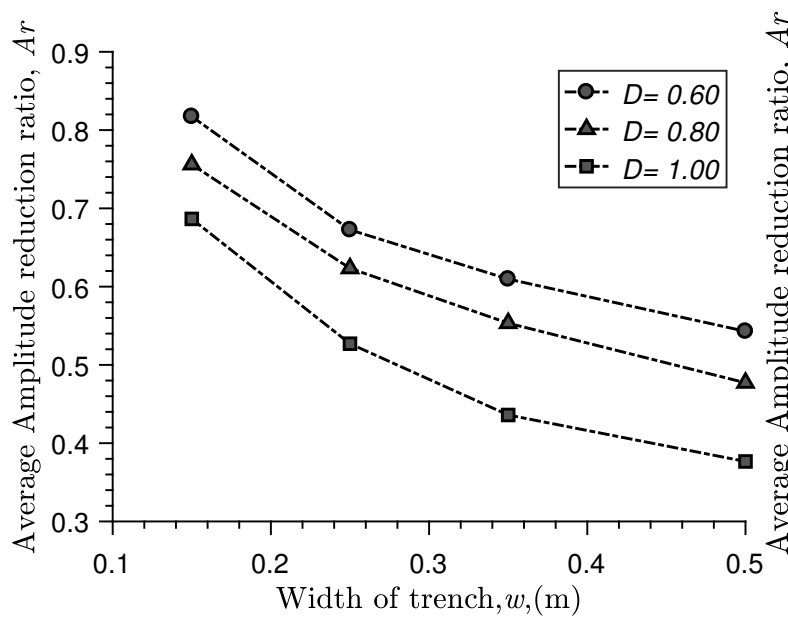

(c) $\operatorname{Ar}$ vs $w, L=1.5$

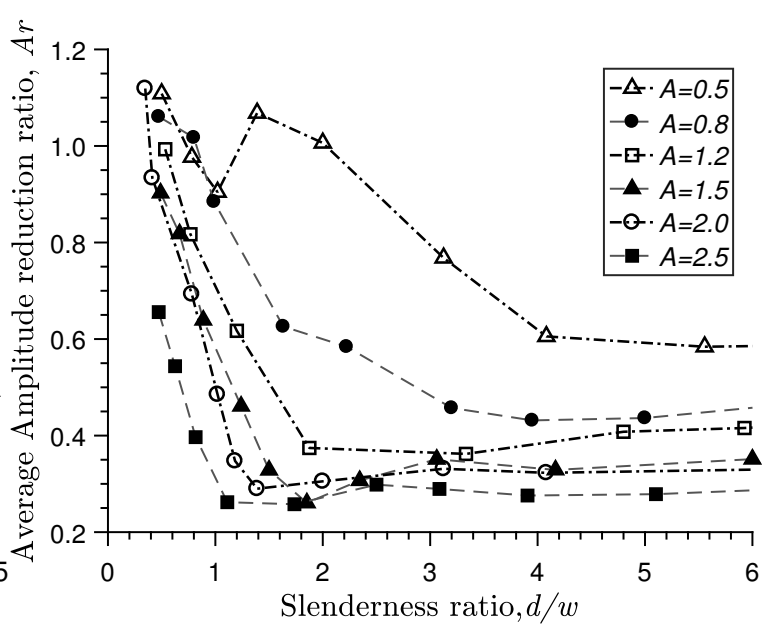

(d) $A r$ vs $d / w$

Figure 7: Variation of average amplitude reduction ratio with change in various geometrical parameters of geofoam trench: (a) normalized screening distance, (b)-(c) width, and (d) slenderness ratio 


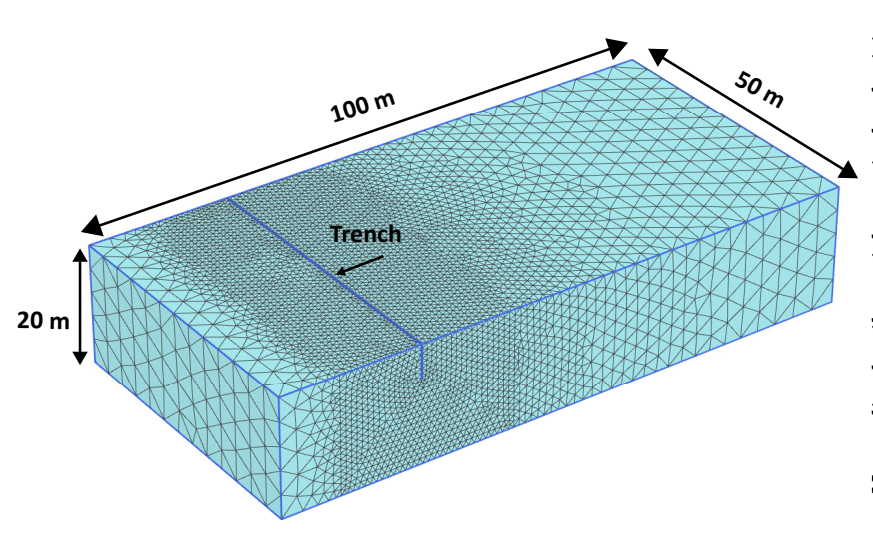

(a) 3-D Model

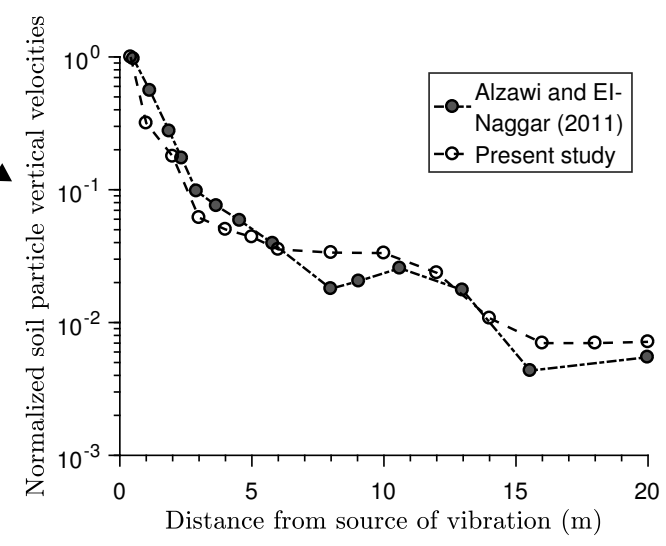

(b) Model validation

Figure 8: 3-D analysis of geofoam trenches (a) typical model developed in PLAXIS, and (b) validation of the numerical model , $l=2.5 \mathrm{~m}, f=50 \mathrm{~Hz}$

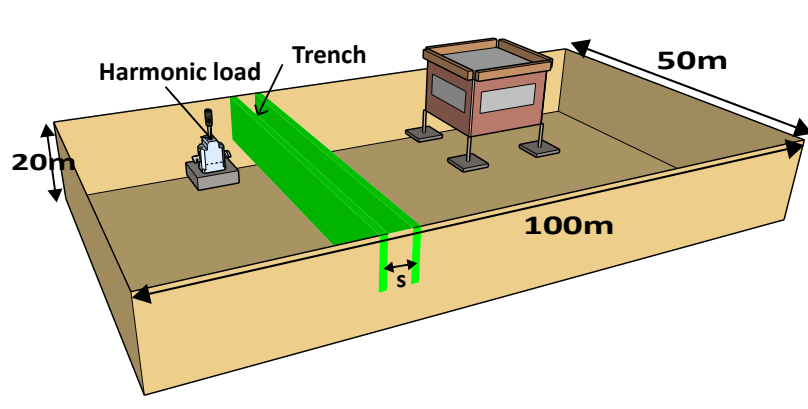

(a) Schematic representation

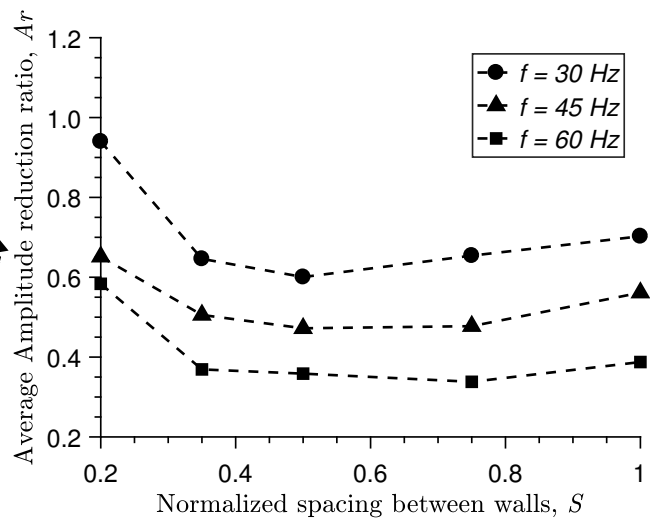

(b) $\operatorname{Ar}$ vs $S$

Figure 9: Analysis of a double walled continuous rectangular geofoam trench system (a) schematic representation of the system, and (b) variation of average amplitude reduction ratio with change in normalized spacing between the two walls 


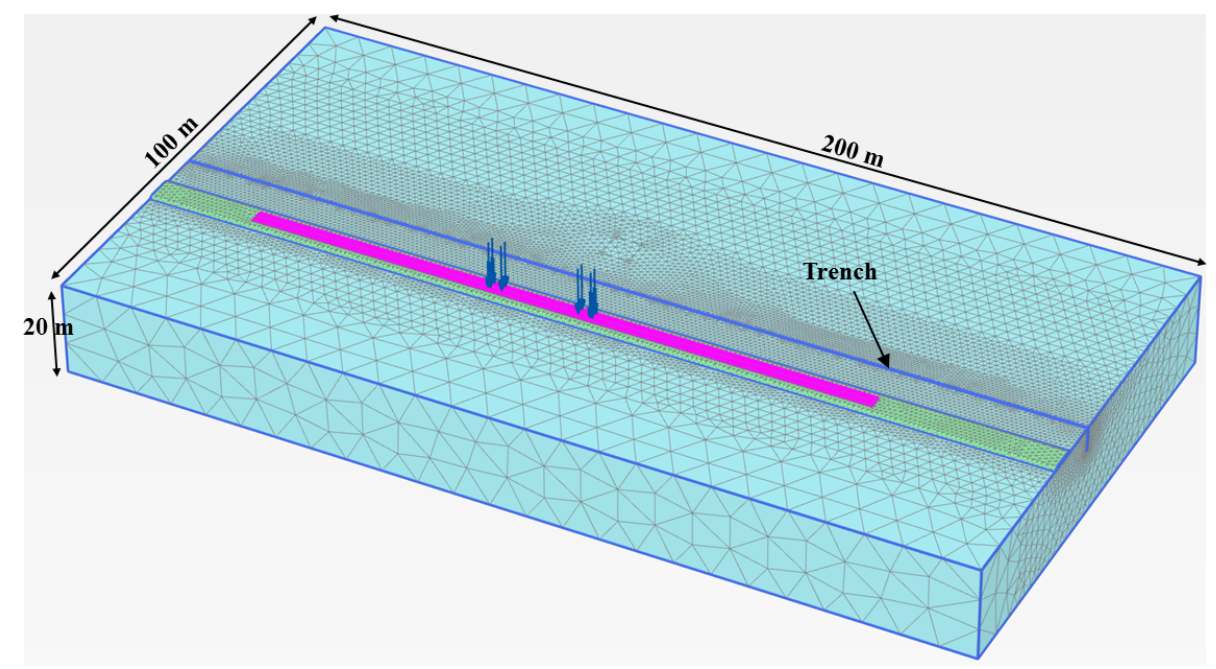

Figure 10: 3-D numerical model developed for simulating moving load

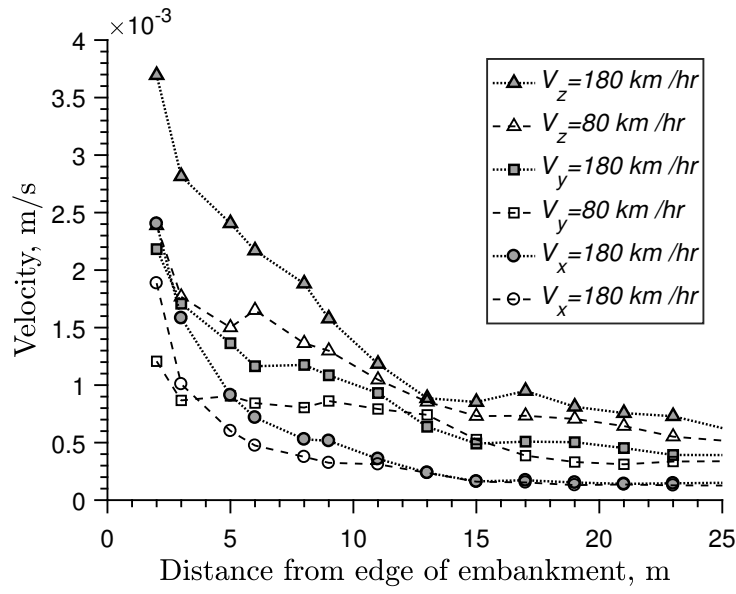

(a)

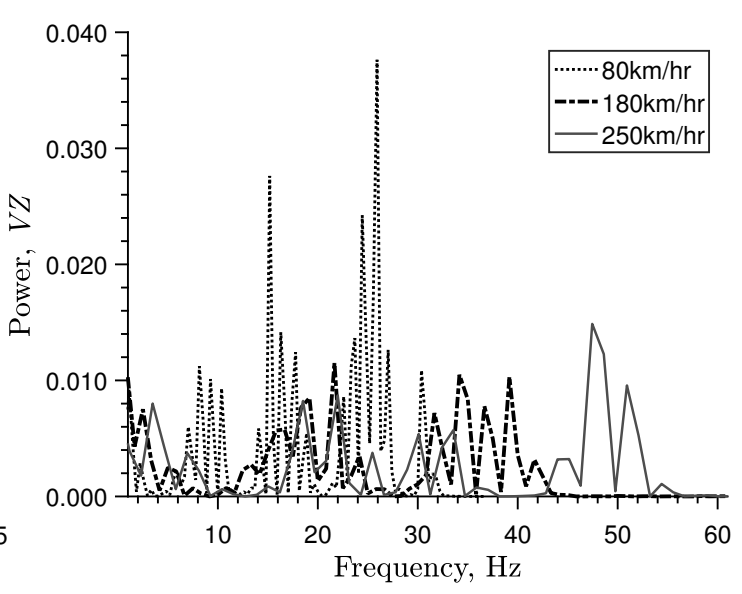

(b)

Figure 11: Variation of velocity of soil particles with change in train speed in absence of trench (a) time domain, and (b) frequency domain 


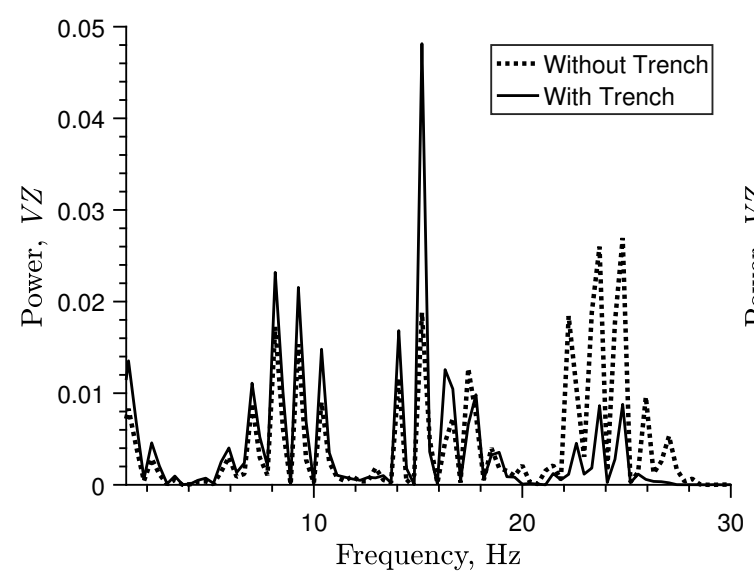

(a) $80 \mathrm{~km} / \mathrm{hr}$

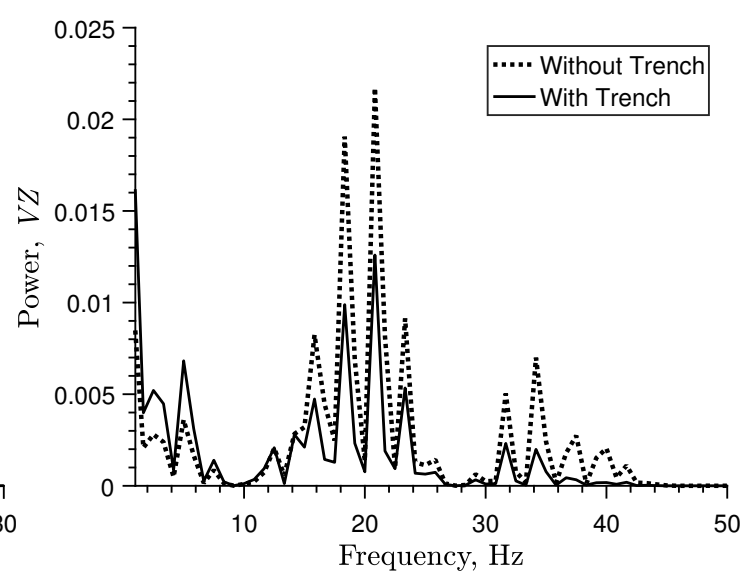

(b) $180 \mathrm{~km} / \mathrm{hr}$

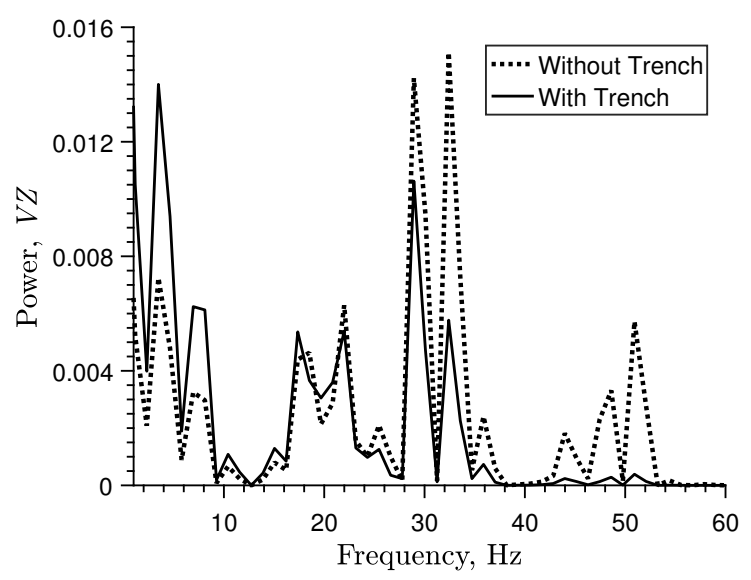

(c) $250 \mathrm{~km} / \mathrm{hr}$

Figure 12: Comparative analysis of velocity in frequency domain in presence and absence of trench (a) $80 \mathrm{~km} / \mathrm{hr}$ (b) $180 \mathrm{~km} / \mathrm{hr}$, and (c) $250 \mathrm{~km} / \mathrm{hr}$ 